\title{
Calibration of Vibrating-Sample Magnetometers
}

\author{
W. E. Case* and R. D. Harrington*
}

\author{
Institute for Basic Standards, National Bureau of Standards, Boulder, Colo.
}

(June 10, 1966)

\begin{abstract}
An evaluation of two of the most widely accepted methods for calibrating vibrating-sample magnetometers is given. The comparison method uses a material of known magnetization such as pure nickel. In the slope method, the magnetometer is calibrated from the low field linear slope of the magnetization curve of a sample of high permeability.

The primary source of error in the comparison method arises from an uncertainty in the absolute magnetization of nickel and its dependence on environmental conditions. The study indicated that better accuracy can be expected from the slope method. The use of pure iron in this method was found preferable to high permeability ferrites.
\end{abstract}

Key Words: Ferro- and ferrimagnetism, magnetization, magnetometer calibration, measurement of magnetization, saturation magnetization, vibrating-sample magnetometer.

\section{Introduction}

The vibrating-sample magnetometer as developed by Foner $[1]^{1}$ is rapidly becoming one of the more commonly accepted techniques for determining the saturation magnetization of ferrimagnetic materials. The many improvements and refinements which have been made in these instruments in recent years have been thoroughly described by Feldmann and Hunt $[2,3,4]$. Although well designed magnetometers of this type prese.tly exist, their ultimate accuracy is still dependent on the calibration technique used in the measurement process. At the present time, two different calibration methods have received the greatest emphasis and have been included in a standard test method for saturation magnetization of nonmetallic magnetic materials by the American Society for Testing and Materials [5]. In the comparison method the magnetometer is calibrated using a material of known magnetization which is usually a sphere of pure nickel. In the slope method the instrument is calibrated from the initial slope of the magnetization curve of a spherical sample of high permeability. It is the purpose of this paper to describe the results of a study of the advantages and disadvantages of each of the above two calibration techniques.

The more commonly used comparison method will first be discussed with a brief description of some of the precautions which we found necessary for improving the accuracy of vibrating-sample instruments in general. The experimental evaluation of the slope method will then be described. The advantages of using ultrapure iron rather than high permeability ferrites which were mentioned [5] for use in the slope method will also be discussed.

* Radio Standards Physics Division, NBS Boulder Laboratories, Boulder, Colo.

${ }^{1}$ Figures in brackets indicate the literature references at the end of this paper.

\section{Comparison Method}

The saturation magnetization of a magnetic material using a Foner type vibrating-sample magnetometer is determined from the voltage generated in a set of pickup coils by a magnetized spherical sample vibrating perpendicular to the applied magnetic field as shown in figure 1 . The voltage generated in these coils is proportional to the magnetization of the sample [1]. We may thus write

$$
E_{0}=K V_{0}\left(4 \pi M_{0}\right),
$$

where $E_{0}$ is the voltage induced in the pickup coils by a sample of magnetization $M_{0}$ and volume $V_{0}$, and $K$ is the proportionality constant. The factor $4 \pi$ has been inserted since the saturation induction, $4 \pi M_{0}$, given

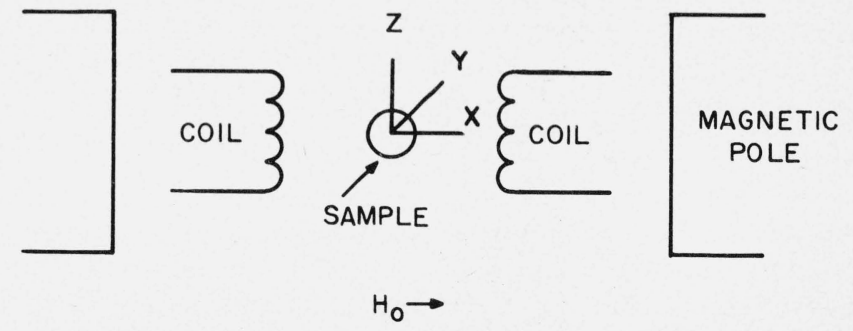

FIGURE 1. Sample and coil arrangement in electromagnet with $\mathrm{x}, \mathrm{y}$, and $\mathrm{z}$ coordinates. 
in gauss, is normally quoted in the literature in describing these materials when using CGS units. In the comparison method, $K$ may be readily obtained from the measured voltage, $E_{s}$, obtained using a standard sample of volume $V_{s}$ and known magnetization $M_{s}$. Thus from eq (1), the magnetization $M_{0}$ of an unknown sample using the comparison calibration method becomes,

$$
4 \pi M_{0}=\left(\frac{E_{0}}{E_{s}}\right)\left(\frac{D_{s}^{3}}{D_{0}^{3}}\right) 4 \pi M_{s},
$$

where the ratio of spherical volumes, $V_{s}$ and $V_{0}$, have been replaced by the ratio of the corresponding cube of the diameters, $D_{s}$ and $D_{0}$.

It is apparent that the accuracy of any measurement depends directly upon how well one knows the magnetization of the standard sample, $M_{s}$. However, before confronting this problem it is desirable to reduce the possible errors from the voltage and diameter terms to a minimum value.

\subsection{Sample Diameter Measurements}

It can be seen that the cube of the diameter of the spheres appears in eq (2) which magnifies any errors in the diameter terms. It is thus necessary to grind highly spherical samples and then measure their average diameter to the highest possible accuracy.

We have found that a sphere grinder such as described by Cross [6] will usually yield spheres that are out of round in the order of only 0.2 percent. Some spheres have been ground better than 0.05 percent out of round, while other spheres ground from a soft material such as nickel may be 0.5 percent out of round. The percentage of out of roundness is

here defined as $100 \frac{\text { max diam }- \text { min diam }}{\text { min diam }}$.

The diameter of the spheres is usually determined from an average of 20 to 30 random measurements made on a given sphere using an electronic comparator calibrated with gage blocks.

In some cases where the deviations in diameter are somewhat larger such as for nickel, it has been found more accurate to determine the volume and average diameter of the sphere from mass and density measurements. This procedure still requires obtaining at least one good spherical sample in order to accurately determine the density. This density is then used in determining the volumes of other less round spheres of the same material assuming the density is constant.

\subsection{Voltage Measurements}

Several factors will of course influence the voltage readings. Repeatability or precision of the voltage readings $E_{0}$ and $E_{s}$ is, of course, of considerable importance. Since the above voltage readings are from two different samples, it is necessary to easily attach and remove each sample from the vibrating rod with the least disturbance possible. In one approach, the sample was glued to a plastic holder which in turn was fastened to the vibrating rod with a plastic screw. Repeatability varied from 0.2 to 1.5 percent. A better approach with a repeatability of less than 0.1 percent is to cement the sample to a small plastic holder which is in turn held on the end of the vibrating rod by means of a vacuum. The samples and corresponding holders are attached or removed from the rod by merely controlling the vacuum.

Another source of error associated with voltage readings is related to the position of the pickup coils with respect to the sample position, as has been described by previous authors $[1,3]$. To study the effects of coil adjustment, the pickup coils in our instrument were mounted on a plastic beam which was fastened to a milling machine table to provide vernier adjustment in the three dimensions shown in figure 1. Experimental data indicate that the coils must be adjusted each time a sample is put in the instrument to within a few thousandths of an inch of the maximum voltage reading in the $y$ and $z$ direction, and a minimum reading in the $x$ direction. For example, a change of 0.010 inch in the $z$ direction gave a change of 0.2 percent in output voltage; a 0.020 inch change gave an 0.8 percent change in output voltage. The distance between the coils from center to center is approximately $1 \frac{1}{8}$ inches.

Still other precautions were found necessary for obtaining accurate voltage readings. (1) The magnetometer was mechanically isolated from the electromagnet. Mounting the vibrating rod system on a wooden table, independently supported by the floor, reduced the residual signal as read on a voltage ratio transformer from 0.0014 to 0.0001 at a field of $10000 \mathrm{Oe}$. (2) The sample pickup coils were mechanically isolated from the electromagnet. Mounting the pickup coils on a floor supported table, independent of the magnet, reduced the residual noise. (3) The empty holder voltage readings were subtracted from the corresponding sample readings. Failure to do this can result in a 0.1 percent error in a typical ratio transformer reading of 0.1000 with an empty holder reading of 0.0001 . (4) A high field was applied to the sample before taking measurements coming down the magnetization curve to avoid any hysteresis ambiguities. This is of greater importance when measuring at low applied fields such as in the slope calibration method as will be discussed later in the paper. (5) The temperature of the magnetometer and sample were stabilized to a few tenths of a degree centigrade during a measurement. This was necessary for holding any variations in the calibration constant to 0.1 percent or less during a measurement. Any further instability effects due to temperature and other sources are reduced by calibrating the system both immediately before and after a measurement is made on an unknown sample and interpolating the calibration constant as a function of time.

Another possible source of error in vibrating-sample magnetometers is related to magnetic image effects in 
the pole caps of the electromagnet. Errors from this situation are apparently reduced by eddy current shielding in the pole caps as pointed out by Foner [1]. Using a 2-inch air gap in the magnet, he estimated the image effect to be less than 0.2 percent. In comparing data on iron samples using a 2 -inch and 3-inch air gap at $9000 \mathrm{Oe}$ applied field, we obtained agreement of better than 0.1 percent which likewise implies that image effects are small.

In addition to the above efforts to avoid errors, a further check on the accuracy of the voltage readings was made by measuring $E$ for several different sized samples of the same material at a given field and comparing the $E / D^{3}$ ratios. In order to avoid errors due to equipment drift, it was found advantageous to refer the $E / D^{3}$ readings of each of the samples to the corresponding $E / D^{3}$ value of one of the samples arbitrarily chosen as a reference $(\mathrm{R})$. The voltage from this reference sample was measured immediately before and after each of the unknown samples and the reference voltage used for calculation was obtained from interpolation between these two values. Disagreement of slightly greater than 0.1 percent was obtained as shown in figure 2 . These results in conjunction with the preceding discussion leads to the conclusion that it is possible to hold any errors in the diameter and voltage terms of eq (2) to less than a few tenths of a percent.

\subsection{Magnetization of Standard Sample}

The saturation magnetization of pure nickel is almost universally used as a standard when ferromagnetic or ferrimagnetic materials are considered. Few investigators have measured the absolute value of $4 \pi M_{s}$ of nickel. The results of a literature search as shown in table 1 indicate several percent variation among those values which have been reported. Most of these values in the literature are given in terms of emu per gram. We have not converted these values to $4 \pi M_{s}$ in gauss since the density of the reported material was not always known. Even if one chooses the correct value, it is difficult to know if his own nickel sample is of the same composition and is being measured under the same conditions that were present when the absolute value was determined. This problem results from the fact that the magnetization of any given sample of nickel depends upon purity, density, magnetic field, temperature, strains, annealing, etc.

The literature contains equations or graphs for correcting for density, temperature, and field effects when the experimental conditions differ from those related to the absolute values [12]. However, some of these corrections may not in themselves be entirely correct. A typical example might be the equation for the approach to saturation which could be used to predict the magnetization of nickel at some other field than where the absolute value is quoted. However, assuming that accurate corrections could be made for temperature, density, and applied field, a problem still exists regarding purity and strain effects.

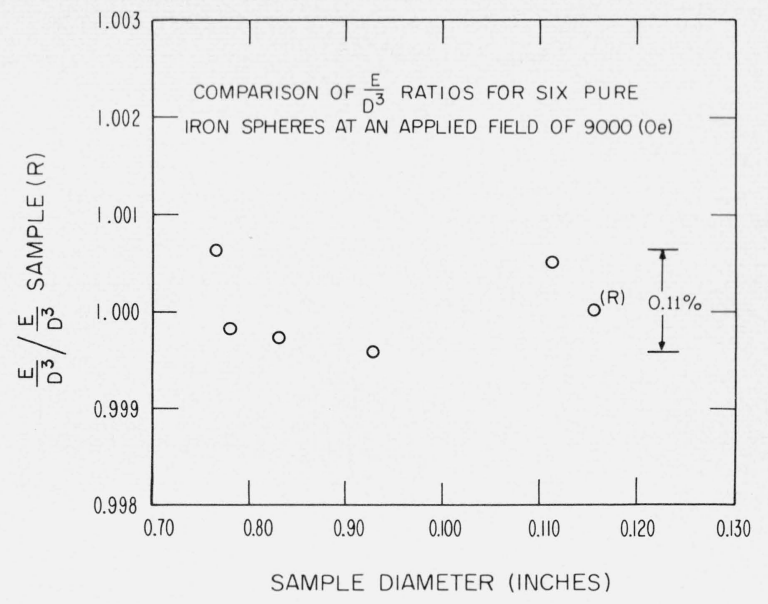

Figure 2. Comparison of $\mathrm{E} / \mathrm{D}^{3}$ ratios of pure iron spheres as a function of sample diameter.

The data were normalized to the $E / D^{3}$ ratio of one of the spheres, arbitrarily chosen as pure iron reference sample, (R).

TABLE 1. Values of saturation moment (emu) per gram for pure nickel

\begin{tabular}{|c|c|c|}
\hline & Ref. & Remarks \\
\hline$e m u / g$ & & \\
\hline 58.1 & [7] & $\begin{array}{l}\text { Single crystal. a Calculated emu. Reference gives } \\
B-H=6500 \text { gauss. Temperature and field not } \\
\text { given. }\end{array}$ \\
\hline $55.5 \pm 1$ & [8] & Temp. $25^{\circ} \mathrm{C}$. Field not given. \\
\hline $55.05 \pm .05$ & [9] & Temp. $15^{\circ} \mathrm{C}$. Extrapolated to infinite field. \\
\hline 54.98 & [10] & $\begin{array}{l}{ }^{*} \text { Calculated from }(B-H) \infty=6150 \text { gauss for density } \\
8.901 \frac{\text { grams }}{\mathrm{cm}^{3} \text { and infinite field }}\end{array}$ \\
\hline $54.73^{\mathrm{b}}$ & [11] & Temp. $20^{\circ} \mathrm{C} . \quad$ Field 18000 (Oe.). \\
\hline $54.55^{\mathrm{b}}$ & [11] & Temp. $20^{\circ} \mathrm{C}$. Field $8000(\mathrm{Oe})$ \\
\hline $54.39^{\mathrm{b}}$ & [12] & Temp. $15^{\circ} \mathrm{C}$. Field not given. \\
\hline $54.27^{\mathrm{b}}$ & [5] & $\begin{array}{l}\text { Room temp. Field not given. }{ }^{\text {a }} \text { Calculated from } \\
B-H=4 \pi M_{s}=6070 \text { gauss and density }=8.90 \frac{\text { rrams }}{\mathrm{cm}^{3}}\end{array}$ \\
\hline
\end{tabular}

${ }^{\text {a }}$ Data in reference given in $B-H$ gauss. From this we calculated emu $=\frac{B-H}{4 \pi \text { density }}$ ${ }^{\mathrm{b}}$ These values are based on Weiss values.

In general, one hopes that his particular sample is of the same degree of purity as the material used in obtaining the absolute values quoted in the literature. The problem of strains can become quite serious as can be seen in figure 3 which shows the magnetization curves obtained before and after annealing four spheres ground from an ultrapure rod of nickel with impurities of less than 0.005 percent. The variation in the data apparently results from small strains induced in the material in the grinding process. Such strains are probably removed by annealing since the curves after annealing are in much better agreement.

The annealing process itself may be a problem since the literature is full of examples of rather large changes in the magnetization curves of nickel due to different annealing treatments. Fortunately such effects do not greatly influence the true saturation value at high fields, but they can become a problem if attempts are made to calibrate an instrument at lower applied fields. 


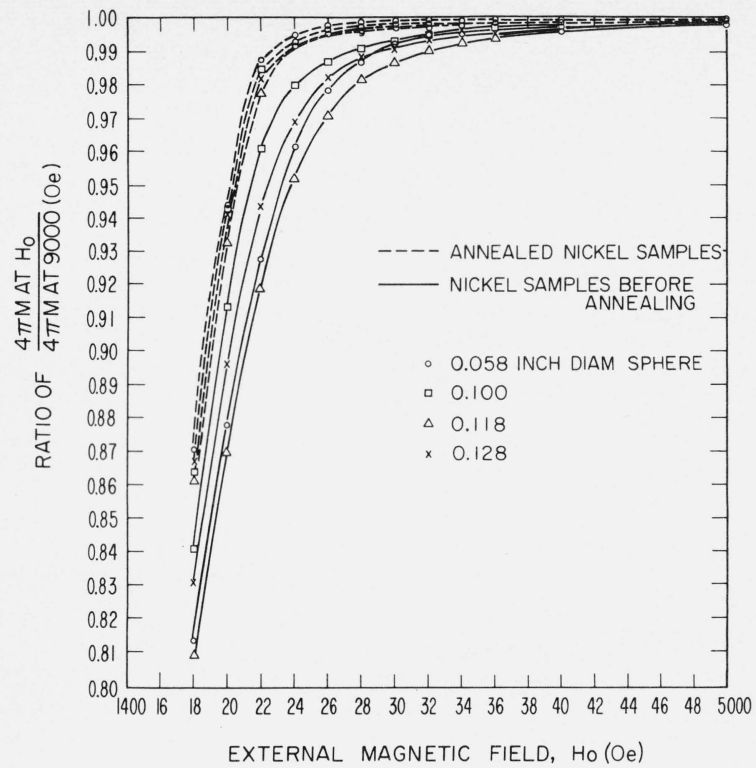

FIGURE 3. Magnetization curves before and after annealing four pure nickel spherical samples.

On the basis of the above arguments, it can be seen that the primary uncertainty in calibrating a vibratingsample magnetometer using the comparison method may well reside in the value of the standard sample. As was shown, the measured diameters of the spheres and corresponding voltages in eq (2) can be determined to a few tenths of a percent. However, unless extreme care is exercised in choosing the value of the standard sample, a further error exceeding 1 percent may be introduced into the problem. In the following section, we will show that the slope method was developed primarily to avoid this problem of uncertainty in $4 \pi M_{s}$ of the standard sample.

\section{Slope Method}

\subsection{Evaluation of Error}

The slope method is based on the observation that the voltage in the pickup coils from a spherical sample is a linear function of the applied field over the lower region of the magnetization curve [13]. Typical curves for a high permeability ferrite and a pure iron splere showing this situation may be seen in figure 4. As shown in the appendix, the slope of these curve $\mathrm{S}$ in the linear region is relatively insensitive to the permeability of the material. It is also shown that this allows the equation for determining the unknown magnetization of a test sample at some field $H$ using the slope method to be written as follows:

$$
4 \pi M_{0}=\frac{E_{0} D_{c}^{3}}{\left[\frac{1}{\mu-1}+N\right] \frac{\Delta E_{c}}{\Delta H_{c}} D_{0}^{3}}
$$

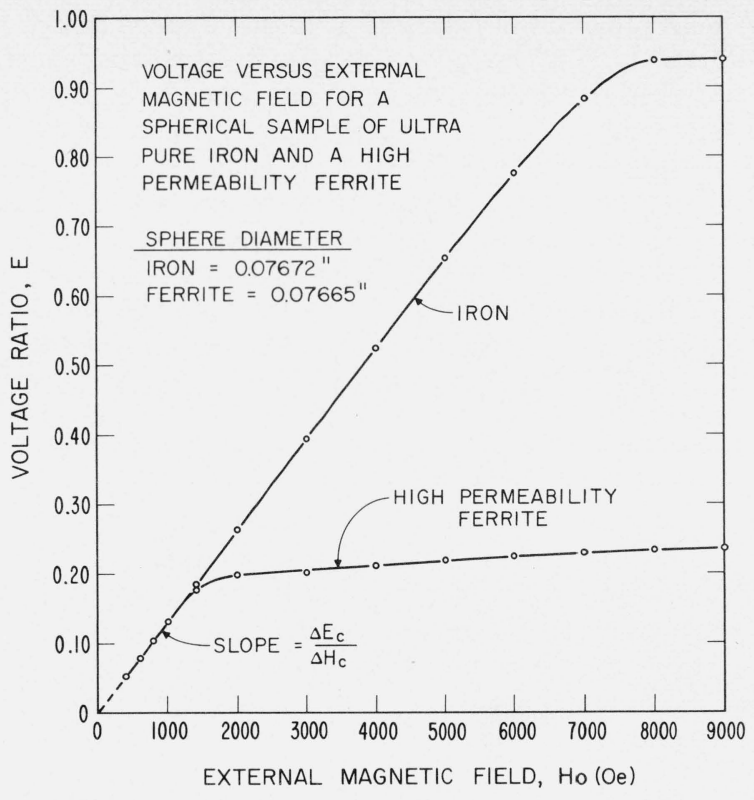

FIGURE 4. Comparison of the linear region of the $\frac{\text { sample voltage }}{\text { reference voltage }}$ ratio curves for an ultrapure iron and a high permeability ferrite sample of equal size.

where $\quad D_{c}=$ diameter of calibrating sphere,

$D_{0}=$ diameter of unknown sample sphere,

$N=$ demagnetizing factor of calibrating sample,

$\mu=$ relative permeability of calibrating sample,

$\Delta H_{c}=$ change in de magnetic field applied to calibrating sample in linear portion of curve (see fig. 4),

$E_{0}=$ coil voltage from unknown sample minus empty holder reading,

$\Delta E_{c}=$ change in coil voltage from calibrating sample corresponding to $\Delta H_{c}$.

For greater precision the two voltage readings used for determining $\Delta E_{c}$, were taken at $H_{c}$ and zero applied field. Although figure 4 implies that the voltage is zero at the origin, a considerably expanded plot of these results shows a small voltage corresponding to a slight remanence at zero applied field is present.

We have already pointed out that an accuracy in the order of 0.1 percent to 0.2 percent is not unreasonable in the voltage and diameter measurements as was shown in figure 2. The three remaining values in eq (3) are the permeability, $\mu$, the demagnetizing factor $N$, and the field, $H_{c}$ used in determining the slope $\Delta E_{c} / \Delta H_{c}$. The problem of comparing the two calibration methods thus reduces to a comparison of the accuracy with which we can determine $\mu, N$, and $H_{c}$ to the accuracy with which we know $4 \pi M_{s}$ of a standard sample as used in the previously described method.

Any errors in the measurement of $\boldsymbol{H}_{c}$ may readily be reduced to a negligible amount by using a nuclear 
resonance gaussmeter in conjunction with a frequency counter. $\Delta E_{c} / \Delta H_{c}$ should be taken for several values of $H_{c}$ to be sure that the linear portion of the curve is being used.

Consider now the remaining quantity $\left(\frac{1}{\mu-1}+N\right)$. The permeability can, of course, have a wide range of values depending upon the type of calibrating sample used. However, as Frederick [13] pointed out the versatility of this method is considerably enhanced by choosing a material of high permeability. Under these conditions, the $\frac{1}{\mu-1}$ term is very small compared with $N=1 / 3$. For example, if we use ferrites whose permeability is at least 2000 , then the difference in assuming $\mu=\infty$ compared to using the initial permeability of 2000 in eq (3) would be only 0.15 percent. Thus for samples with permeabilities of 2000 or greater, the error contributed by uncertainties in the permeability term should be less than this. The fact that we do not need to know $\mu$ accurately if it is sufficiently high in this slope method is the primary advantage of this technique over the comparison method. Furthermore, materials having permeabilities greater than 2000 are readily available and may easily be ground into spherical shapes.

In the case of a perfect sphere, $N=1 / 3$; however, any deviations from a true sphere would cause an error in $N$ which would cause practically the same error in eq (3). The effect of out of roundness can be calculated, theoretically, if we assume that the out of round sample is still a prolate or oblate spheroid. Stoner [14] has shown that for the nearly spherical prolate spheroid with $a>b, \epsilon^{2}=1-\left(\frac{b}{a}\right)^{2} \ll 1$

$$
\begin{aligned}
& N_{p}=\frac{1}{3}-\frac{2}{15} \epsilon^{2}\left(1+\frac{3}{7} \epsilon^{2} \ldots\right) \\
& N_{q}=\frac{1}{2}\left(1-N_{p}\right)=\frac{1}{3}+\frac{1}{15} \epsilon^{2}\left(1+\frac{3}{7} \epsilon^{2} \ldots\right)
\end{aligned}
$$

and for the nearly spherical oblate spheroid with

$$
\begin{gathered}
a<b, \epsilon^{2}=1-\left(\frac{a}{b}\right)^{2} \ll 1 \\
N_{p}=\frac{1}{3}+\frac{2 \epsilon^{2}}{15}\left(1+\frac{4}{7} \epsilon^{2}+\ldots\right) \\
N_{q}=\frac{1}{2}\left(1-N_{p}\right)=\frac{1}{3}-\frac{1}{15} \epsilon^{2}\left(1+\frac{4}{7} \epsilon^{2}+\ldots\right)
\end{gathered}
$$

where $a=$ the polar semi-axis,

$$
b=\text { the equatorial semi-axis, }
$$

$N_{p}=$ demagnetizing factor along the polar axis,

$N_{q}=$ demagnetizing factor along the equatorial axis.

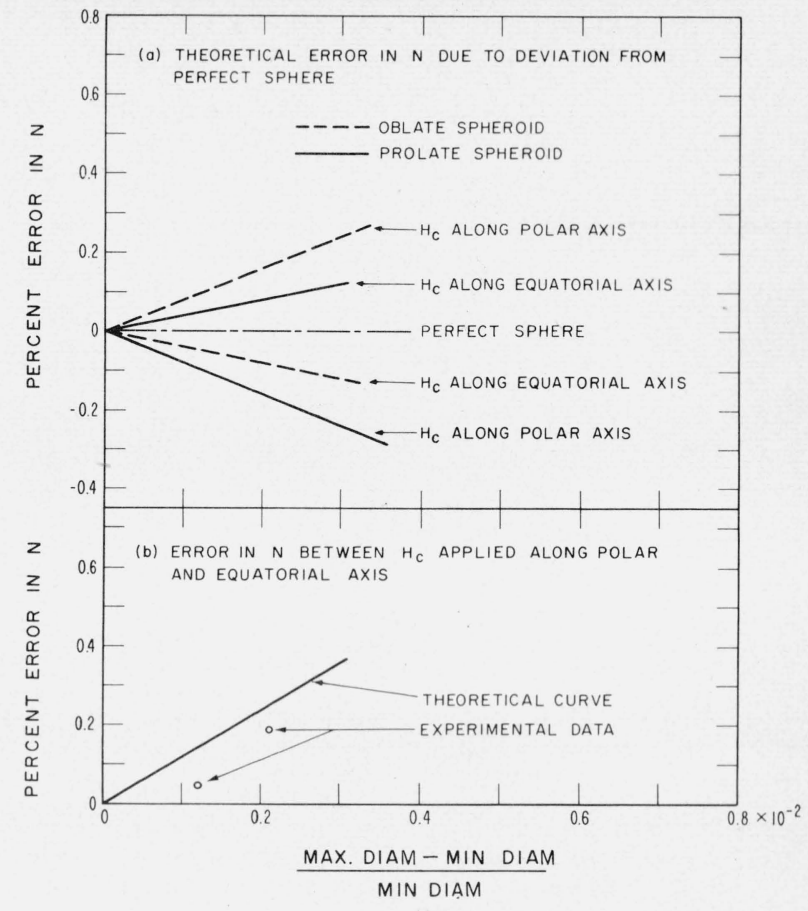

FigURE 5. (a) Theoretical effect of small eccentricity, $\epsilon$, on values of $\mathrm{N}$ when magnetic field, $\mathrm{H}_{\mathrm{c}}$, is applied first along the polar axis and then along the equatorial axis for oblate and prolate spheriods.

(b) Total percent error in $\mathrm{N}$ expected between field along polar axis and along equatorial axis.

(Data are obtained from the difference between corresponding curves in graph (a)). Ex perimental points represent percent error in voltage readings which are proportional to percent error in $N$.

Equations (4), (5), (6), and (7) are shown graphically in figure $5 \mathrm{a}$ where

$$
\frac{\epsilon^{2}}{2} \approx \frac{\text { Max diam }- \text { Min diam }}{\text { Min diam }}
$$

As an experiment, $E_{c}$ was measured along the polar axis and compared with $E_{c}$ measured along the equatorial axis for two samples. The error (difference) in $E_{c}$ as shown in figure $5 \mathrm{~b}$, which is proportional to the corresponding error in $N$, was less than the theoretical curve predicted by taking differences in $N$ in figure $5 \mathrm{a}$. It was concluded that the calibrating spheres should not be out of round more than approximately 0.1 percent. The errors due to sample volume determination are actually greater than those due to misorientation. The diameter measurement has already been discussed earlier in the paper.

The above arguments thus imply that the error to be expected from the $\Delta H_{c}, \mu$, and $N$ terms in eq (3) should be less than a few tenths percent. This result in conjunction with the 0.1 to 0.2 percent possible error in the voltage and diameter terms makes it feasible to expect errors of less than 0.5 percent in using eq (3). 


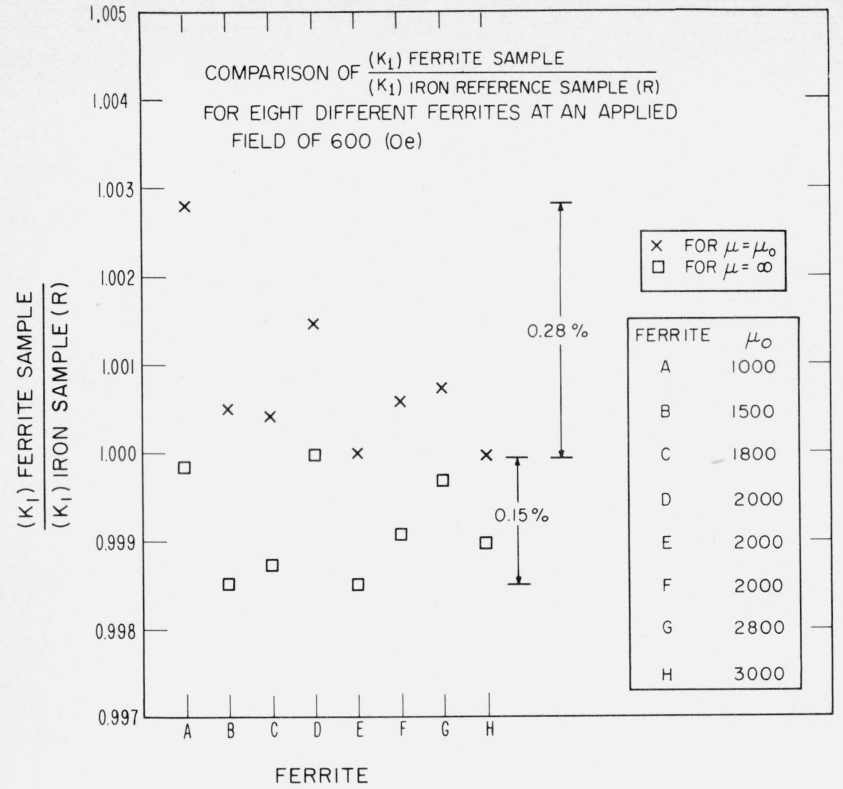

FIGURE 6. Comparison of $\mathrm{K}_{1}$ ratios for several different ferrite samples with initial permeabilities ranging from 1000 to 3000 .

The data were normalized to the arbitrarily chosen pure iron reference sample (R) used in figure 2 .

\subsection{Experimental Results Using High Permeability Ferrites}

In order to further confirm the above analysis, an experimental investigation of the validity of the calibration constant obtained using the slope method was carried out. The calibration constant may be readily obtained by writing eq (3) in the form

$$
4 \pi M_{0}=\frac{1}{K_{1}} \frac{E_{0}}{D_{0}^{3}}
$$

where $K_{1}$ is given by

$$
K_{1}=\frac{\left(\frac{1}{\mu-1}+N\right) \Delta E_{c}}{D_{c}^{3} \Delta H_{c}} .
$$

On the basis of the previous discussion, it appears reasonable that we may set $N=1 / 3$ in this equation with negligible effect on the accuracy. With this assumption, the value of $K_{1}$ as a calibration constant was checked in the following ways:

(1) For a given calibration field $H_{c}, K_{1}$ was compared for several ferrite samples of different size from the same high permeability material ( $\mu$ assumed constant). For six samples ranging from 0.090 to 0.128 -inch diameter, agreement was within 0.15 percent. No size effect trend could be detected.

(2) For a given calibration field, $K_{1}$ was compared for several samples of nearly the same size but for several different ferrite materials with initial permeabilities, $\mu_{0}$, that range from 1000 to 5000 . As was previously discussed, we used an arbitrary reference sample before and after each measurement to improve the accuracy. Using known values of initial permeability $\left[\mu_{0}\right.$, in eq (9)], the variation in the $K_{1}$ ratios as shown in figure 6 for eight high permeability ferrite samples was 0.28 percent. Assuming $\mu=\infty$, the variation was 0.15 percent. This implies that the effective permeability is equal to or greater than the initial permeability as specified by the manufacturer as shown in figure 6. A ninth sample with the highest initial permeability (5000) gave 0.5 to 0.85 percent higher values for $K_{1}$.

(3) For a given sample, $K_{1}$ was compared for several values of the field $H_{c}$. In the 350 to $600 \mathrm{Oe}$ range, $K_{1}$ varied less than 0.1 percent and there was no definite trend as to field dependence. The above procedure was repeated for several calibration samples of different materials of high permeability with similar results.

\subsection{Experimental Results Using Ultrapure Iron}

Because of the discrepancy caused by the highest initial permeability (5000) sample and because of the above 0.3 percent variation of the other ferrites, further investigation was carried out. As can be seen, an ideal calibrating material would be one with a high initial permeability of 10,000 or greater in which case the term, $\frac{1}{\mu-1}$, in eq (9) could be dropped with negligible error. Data given in the literature, $[15,16]$, indicate that high purity iron can be produced with initial permeabilities in this range.

A commercially available sample of high purity polycrystalline iron was obtained with a total impurity of 0.005 percent including less than 0.001 percent carbon impurity. Six samples were ground down to various sizes and measured with the results shown in figure 7. Again iron reference sample $(R)$ was used. The less than 0.1 percent variation in $K_{1}$ for iron shown in the figure is less than the 0.15 percent variation observed between different diameter samples of a given ferrite as mentioned in the previous section as well as the variation observed between different ferrites as shown in figure 6 .

It can be seen from figure 7 , that the iron reference sample $(R)$ is fairly representative of the iron samples. The data in figure 6 were taken using the same iron reference sample $(\mathrm{R})$. Therefore, figure 6 is a means of comparing how well several ferrites with different permeabilities compare with a representative iron sample using $\mu=\mu_{0}$ and $\mu=\infty$ for the ferrites and $\mu=\infty$ for iron in eq (9). It appears the correct $\mu$ value to use for the ferrites lies between $\mu_{0}$ and $\infty$. Some additional ultrapure iron samples were ground, annealed, and reground. Measurements comparing annealed and unannealed ultrapure iron samples agreed within 0.1 percent at applied fields of $600 \mathrm{Oe}$ and 12,000 Oe.

The close agreement in the above results indicates that high purity iron has several advantages when used in the slope method to calibrate a vibrating sample magnetometer. These are (1) high purity iron is 


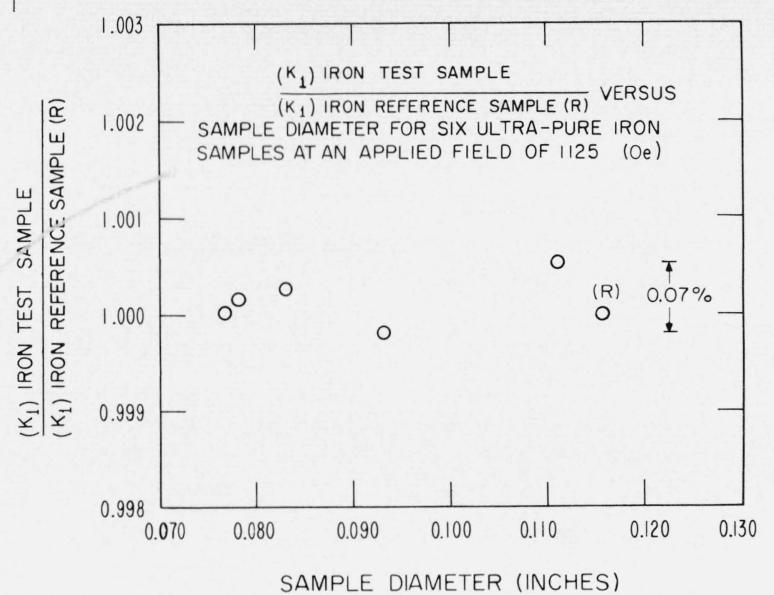

Figure 7. Comparison of $\mathrm{K}_{1}$ ratios for six ultranure iron spheres versus sample diameter for an external field of $112.5(\mathrm{Oe})$.

The data were normalized to one of the spheres, arbitrarily cfosen as pure iron reference sample, $(\mathrm{R})$.

readily available commercially; (2) it is easier to accurately calculate $K_{1}$ since $\mu$ may be assumed to be infinity; (3) the properties of a pure material such as iron are more uniform than ferrites as to porosity, density, homogeneity, etc., which give a more constant $K_{1}$; and (4) because the slope of the linear portion of the iron curve in figure 4 at an applied field of 5000 Oe deviates only 0.5 percent or less from the slope of the curve at $600 \mathrm{Oe}$, a pure iron sphere could be used to calibrate a magnetometer, with some sacrifice in accuracy, at any field up to 5000 Oe using eq (9).

It is apparent that the saturation magnetization of a given spherical sample of pure iron at high fields may readily be determined using the same spherical sample to calibrate the instrument at low fields using the slope method. This self-calibration procedure avoids diameter and sample position errors which may be present when the calibration sample and the sample under test are different. A measurement of the saturation magnetization of iron in this manner provides another means of checking the accuracy of the magnetometer since this quantity may be compared with values given in the literature which are in good agreement. Using this approach, we found that the saturation magnetization data taken on five pure iron samples agreed to within 0.3 percent of measurements taken by Weiss and Forrer [17] and Danan [9]. We measured a saturation magnetization value of $21465 \pm 20$ gauss for a pure iron sphere at $23.3^{\circ} \mathrm{C}$ and an external field of 9000 Oe.

\section{Conclusions}

Experimental observations using the slope calibration technique show that only a few tenths of a percent variation in $K_{1}$ may be expected. Ultrapure iron and most high permeability ferrites can be used for calibration. However, ultrapure iron is preferred for several reasons as noted above. In an actual measurement of $M_{0}$, the additional error in $D_{0}$ and $E_{0}$ would also be present. These errors as discussed earlier in the paper are of the order of one-tenth to a few tenths of a percent. These results thus show that an accuracy of better than 0.5 percent for magnetization measurements can easily be obtained with the slope method.

In the comparison method previously discussed, we had the same one-tenth to a few tenths percent error in $E_{0}$ and $D_{0}$. In addition, we had the error due to the uncertainty in $4 \pi M_{s}$ of the standard nickel sample. It is possible that this might be known to a few tenths of a percent which would make this method comparable to the slope technique. However, the sensitivity of nickel to its environment and the uncertainty in the true absolute value make this seem unlikely. It thus appears that the slope method is preferable to the comparison technique for calibrating vibratingsample magnetometers.

The authors express their thanks to Nolan Frederick who designed and built the vibrating-sample magnetometer and to William McNaney for precision measurements of the specimen diameters.

\section{Appendix. Derivation of Equation (3)}

The relationship between the magnetization $M$ and internal field, $H_{i}$, of a material in the demagnetized state is defined as [18]

$$
M=\frac{\mu-1}{4 \pi} H_{i},
$$

where $\mu$ is the relative permeability. The internal field, $H_{i}$, in a finite specimen is related to the external field $H_{0}$ by means of the demagnetizing factor $N$ as follows: [19]

$$
H_{i}=H_{0}-N 4 \pi M \text {. }
$$

Substituting this value of $H_{i}$ in the first equation and solving for $4 \pi M$, we get

$$
4 \pi M=\frac{H_{0}}{\frac{1}{\mu-1}+N} .
$$

For a calibrating sample, this equation may be written

$$
4 \pi M_{c}=\frac{H_{c}}{\frac{1}{\mu-1}+N} .
$$

As in eq (1) in the text, we have

$$
E_{c}=K V_{c} 4 \pi M_{c}
$$




\section{References}

$$
\Delta E_{c}=K V_{c} \Delta 4 \pi M_{c}=\frac{K V_{c} \Delta H_{c}}{\frac{1}{\mu-1}+N}
$$

and thus

$$
K=\frac{\left(\frac{1}{\mu-1}+N\right) \Delta E_{c}}{V_{c} \Delta H_{c}} .
$$

For an unknown sample, $E_{0}=K V_{0} 4 \pi M_{0}$, as in eq (1) in the text,

$$
4 \pi M_{0}=\frac{E_{0}}{K V_{0}}
$$

Substituting the value of $K$,

$$
4 \pi M_{0}=\frac{E_{0} V_{c}}{\left[\frac{1}{\mu-1}+N\right] \frac{\Delta E_{c}}{\Delta H_{c}} V_{0}}
$$

or expressing the volumes in terms of diameter, we obtain eq (3) in the text.

$$
4 \pi M_{0}=\frac{E_{0} D_{c}^{3}}{\left[\frac{1}{\mu-1}+N\right] \frac{\Delta E_{c}}{\Delta H_{c}} D_{0}^{3}} .
$$

It should be noted that the above derivation is based on the assumption that the sample is in the demagnetized state. It was previously mentioned that a very small voltage due to a slight remanent magnetization may be observed at zero applied field in the spherical samples. However, the excellent agreement in the data obtained for different materials implies that eq (3) is still essentially valid even though a slight hysteresis exists in the samples.
[1] S. Foner, Versatile and sensitive vibrating-sample magnetometer, Rev. Sci. Inst. 30, No. 7, 548-557 (1959).

[2] D. Feldmann and R. Hunt, Konstruktionsgrundlagen der Proben-Vibrationsmagnetometer, Z. Instr. 72, Heft 9, 313318 (1964).

[3] D. Feldmann and R. Hunt, Vibrationsmagnetometer, Uberblick und Stand der Entwicklung, Z. Instr. 72, Heft 9, 259-266 (1964).

[4] D. Feldmann and R. Hunt, Schaltungstechnik der Probenvibrationsmagnetometer, Z. Instr. 73, Heft 3, 62-65 (1965).

[5] ASTM Test Method C 527-63T. Saturation Magnetization or Saturation Induction of Nonmetallic Magnetic Materials.

[6] P. Cross, Instrument for making individual spheres to a high degree of accuracy, Rev. Sci. Instr. 32, No. 11, 1179-1181 (1961),

[7] E. Wise and R. Schaefer, Properties of Pure Nickel, Metals and Alloys, 16, 424 (1942).

[8] P. Selwood, Magnetochemistry, p. 38, (Interscience Publishers, Inc., New York 1956).

[9] H. Danan, Magnetism-study of the variation of magnetization of pure polycrystalline iron and nickel in the vicinity of saturation, Comptes Rendus 246, 73 (1958).

[10] L. Jordan and W. Swanger, The properties of pure nickel (Magnetic Properties, R. Sanford) J. Res. NBS 5, 1303 (1930).

[11] International Critical Tables of Numerical Data Physics, Chemistry and Technology, Vol. VI, p. 403 (McGraw-Hill Book Company, Inc., New York, N.Y., 1929).

[12] R. Bozorth, Ferromagnetism, p. 270 (D. Van Nostrand Company, Inc., Princeton, N.J., 1951).

[13] N. Frederick, A simple calibration technique for vibrating sample and coil magnetometers, Proc. IRE 49, No. 9, 1449 (1961).

[14] E. Stoner, The demagnetizing factors for ellipsoids, Phil. Mag. 7, 36, 803-821 (1945).

[15] R. Bozorth, ibid, pp. 19, 870.

[16] A. Morrish, The Physical Principles of Magnetism, p. 410 (John Wiley \& Sons, Inc. New York City, N.Y.).

[17] P. Weiss and R. Forrer, LaSaturation Absolute Des Ferromagnetiques Et Les Lois D'Approche En Fonction Du Champ Et De La Temperature, Ann. Phys. Paris 12, p. 311 (1929).

[18] R. Bozorth, ibid, p. 5.

[19] R. Bozorth, ibid, p. 10.

(Paper 70C4-236) 